\title{
Caesarea Mauretaniae
}

Ph. Leveau

\section{OpenEdition}

Journals

Édition électronique

URL : http://journals.openedition.org/encyclopedieberbere/1899

DOI : 10.4000/encyclopedieberbere.1899

ISSN : 2262-7197

\section{Éditeur}

Peeters Publishers

\section{Édition imprimée}

Date de publication : 1 septembre 1992

Pagination : 1698-1706

ISBN : 2-85744-581-4

ISSN : 1015-7344

\section{Référence électronique}

Ph. Leveau, "Caesarea Mauretaniae », Encyclopédie berbère [En ligne], 11 | 1992, document C06, mis en ligne le 01 avril 2013, consulté le 25 septembre 2020. URL : http://journals.openedition.org/ encyclopedieberbere/1899; DOI : https://doi.org/10.4000/encyclopedieberbere.1899

Ce document a été généré automatiquement le 25 septembre 2020

(c) Tous droits réservés 


\title{
Caesarea Mauretaniae
}

\author{
$\mathrm{Ph}$. Leveau
}

1 Le petit port de Cherchel en Algérie occupe l'emplacement de Caesarea de Maurétanie qui fut dans l'antiquité romaine une des plus importantes villes d'Afrique du Nord. Héritière de Iol, qui fut peut-être une des capitales du roi numide massyle, Micipsa, puis du maure Bocchus, elle fut rebaptisée Caesarea par le roi Juba II, le fils de Juba I ${ }^{\text {er }}$, l'allié de Pompée, qu'Auguste plaça en 25 av. J.-C. à la tête du royaume maurétanien restauré. Ptolémée succéda à son père Juba en 23 ap. J.-C. ; mais en 40 il fut éliminé par Caligula et Caesarea devint naturellement la capitale de la nouvelle province de Maurétanie Césarienne créée par l'empereur Claude. Elle devait garder cette fonction de capitale provinciale jusqu'à la fin de l'Antiquité. Si au XVI siècle, les Turcs faillirent un moment lui rendre son rôle de capitale, ce n'était plus durant tout le Moyen-Age qu'une bourgade d'importance secondaire et les historiens arabes la donnent même comme disparue. Sa renaissance moderne date de l'installation des Andalous chassés d'Espagne.

2 Située à une centaine de kilomètres à l'ouest d'Alger, la ville est installée sur un étroit plateau littoral dominé par une zone montagneuse l'isolant au sud des plaines du Chélif. Cette zone montagneuse large d'une trentaine de kilomètres est composée de deux unités : l'Atlas de Cherchel, large de 7 à $8 \mathrm{~km}$ et culminant aux environs de $600 \mathrm{~m}$, et l'Atlas du Bou-Maad dont la crête s'allonge entre 1200 et $1400 \mathrm{~m}$. Une zone synclinale qui constitue le prolongement de la Mitidja, les sépare. Au nord-est, un massif de forme grossièrement ovale (11 km de long sur $8 \mathrm{~km}$ de large), le Chénoua, qui culmine à $904 \mathrm{~m}$, l'isole de la plaine de la Mitidja.

3 Les débuts de l'occupation du site sont encore mal connus. Dans une brève note consacrée à la préhistoire de Cherchel, le Dr H. Marchand observait que, sur ce site, l'importance des vestiges romains a « fait complètement oublier une histoire beaucoup plus ancienne, la préhistoire de la région. C'est ainsi que $S$. Gsell, très averti cependant des choses préhistoriques, ne trouve à citer dans son Atlas archéologique de l'Algérie, aucun travail de préhistoire sur la région ». La situation n'a guère évolué depuis l'année 1932 quand il écrivait ces lignes. Par contre nous sommes maintenant mieux renseignés sur les origines de l'habitat urbain. Celui-ci commence avec la ville d'Iol qui figure sur 
la liste des comptoirs énumérés par le Pseudo-Scylax. Ce nom se rattache à la série des toponymes puniques débutant par le préfixe « 1 » qui signifie île, et c'est précisément dans l'îlot qui, dès l'époque antique, fut rattaché à la côte par une digue, que l'on a d'abord trouvé les premiers vestiges d'une occupation remontant au ve siècle av. J.-C. L'agglomération était sans doute déjà étendue à une époque très ancienne : les fouilles entreprises par une mission algéro-britannique, au centre de la ville moderne, ont révélé, sous un dallage d'époque sévérienne, des niveaux d'occupation beaucoup plus anciens. On ne sait pas encore très bien à quel moment loi accéda au rang de capitale. Ce fut peut-être dès l'apparition d'un royaume de Maurétanie occidentale : on est en effet tenté de mettre en rapport son développement urbain, son rôle bien attesté de capitale à la fin du $\mathrm{I}^{\mathrm{er}}$ siècle av. J.-C, avec le mausolée royal voisin connu sous le nom de Tombeau de la Chrétienne.

Mais quelle que soit l'importance de la ville libyco-punique d'Iol, elle est sans rapport avec celle de la Caesarea refondée par Juba II après qu'il eut reçu d'Auguste une partie des États qu'avaient possédés ses ancêtres. Strabon nous apprend que «l'ancienne ville de loi » fut «rebâtie par Juba, le père de Ptolémée qui changea son nom en celui de Caesarea » (Géographie, 16, 3, 12). La nouvelle Caesarea était une ville romaine, bâtie à la romaine par un prince client accomplissant un acte politique conforme à sa situation, comme le dit explicitement Suétone dans la Vie d'Auguste: "Les rois amis et alliés fondèrent chacun dans son royaume des villes portant le nom de Caesarea " (Vie d'Auguste, 60). Alors que l'on doit attribuer à loi une surface correspondant au maximum à celle de la Cherchel du xix ${ }^{\mathrm{e}}$ siècle, 8 ha, Caesarea est entourée d'un rempart qui enclôt une superficie énorme de 370 ha: son enceinte est une des plus vastes du monde romain.

Caesarea de Maurétanie. L'enceinte antique et la zone effectivement construite à l'époque romaine.

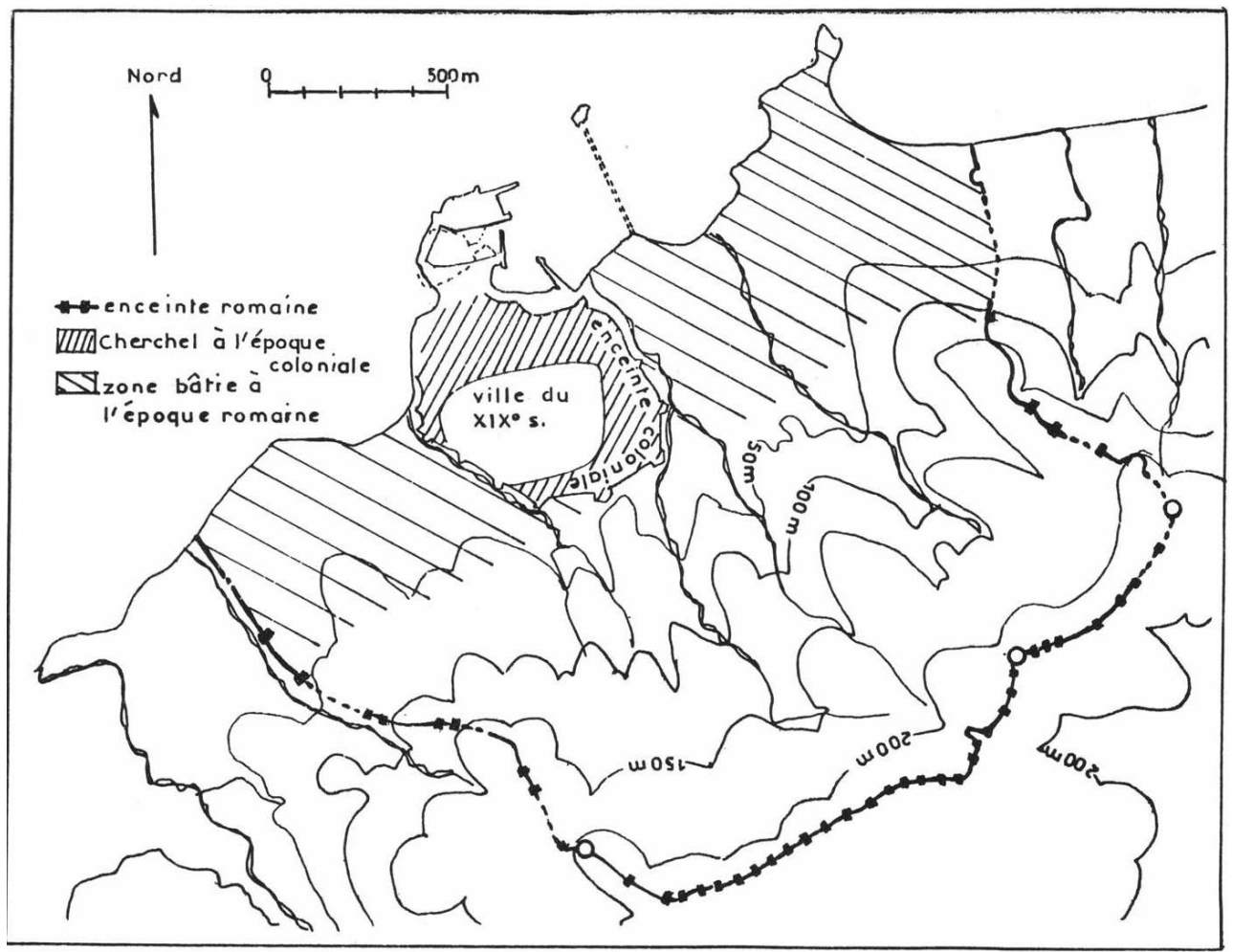


5 La ville disposa du jeu complet des édifices qui caractérisent la ville romaine : ensembles monumentaux liés à la vie politique (forum et bâtiments annexes), thermes, édifices de spectacles (théâtre, amphithéâtre, cirque), aqueduc. Ces édifices s'intègrent dans un plan régulier dont certains éléments caractéristiques ont été mis en évidence. Il est maintenant possible d'ébaucher une histoire du développement urbain de Caesarea.

Plan restitué de Ceasarea de Maurétanie

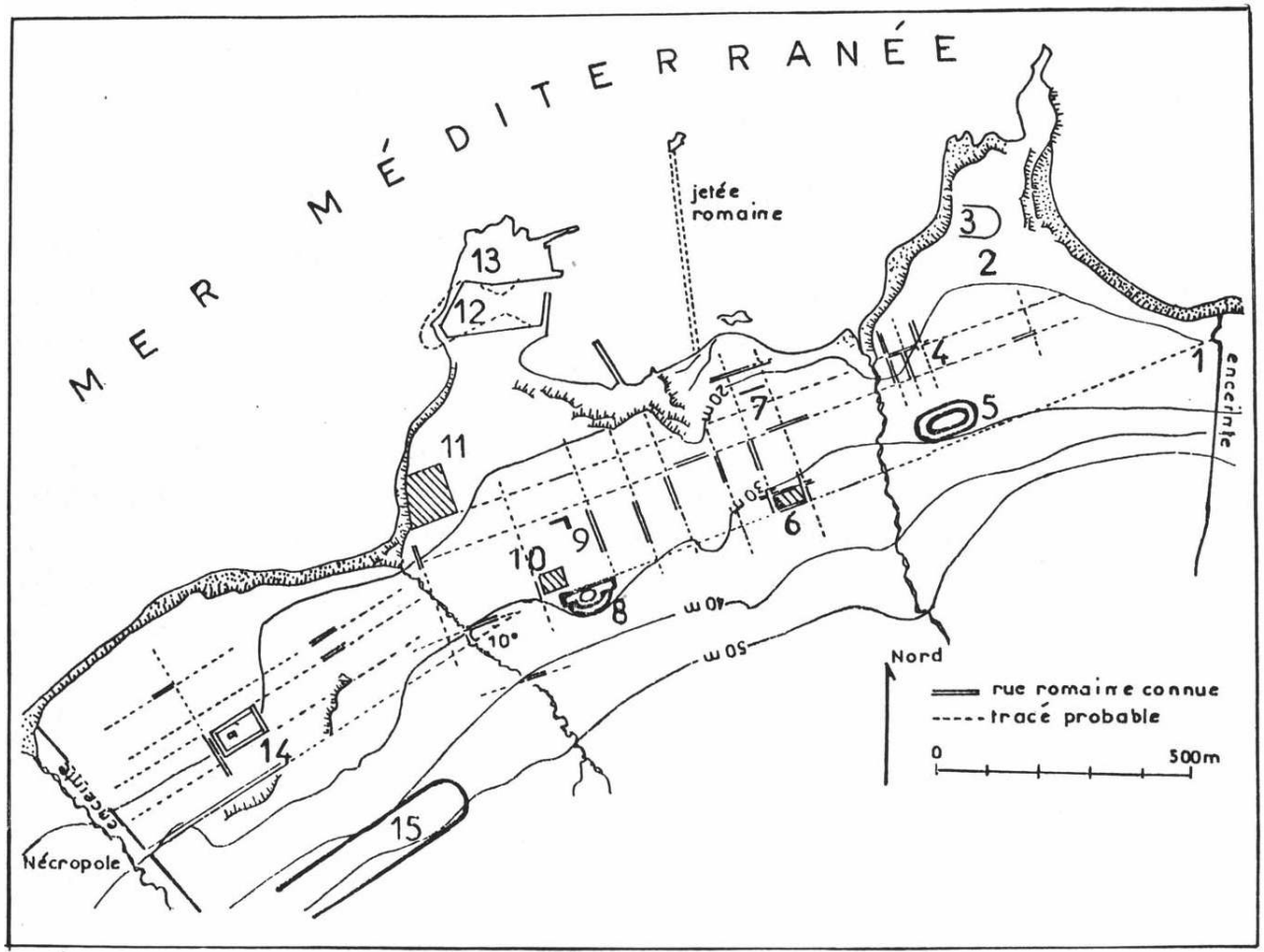

1 : porte principale ; 2 : nécropole pré-romaine ; 3 : stade (?) ; 4 : quartier dégagé en $1958 ; 5$ :

l'amphithéâtre ; 6 : thermes de l'Est ; 7 : temple ; 8 : théâtre ; 9 : forum sévérien ; 10 : thermes du Centre ; 11 : thermes de l'Ouest ; 12 : port militaire antique ; $13:$ phare ; $14:$ quartier ouest dégagé en $1960-1961 ; 15$ : cirque.

6 Confirmant une hypothèse remontant à S. Gsell, les archéologues ont mis en évidence l'origine augustéenne ou disons, pour rendre à Juba ce qui lui revient, jubéenne de cet urbanisme. G. Ch. Picard l'a montré pour le théâtre : il est même l'un des plus anciens d'Afrique, à l'exception de celui d'Utique et daterait du règne d'Auguste. Une étude du plan très particulier de l'amphithéâtre conduit à supposer que le tracé surprenant de son arène (un espace central rectangulaire prolongé sur les petits côtés de deux espaces semi-circulaires) n'est pas dû à la maladresse d'architectes provinciaux. C'est en fait un des rares jalons construits en dur qui permette de suivre l'élaboration du plan classique de l'amphithéâtre.

$7 \quad$ Il a été conçu pour un type de spectacle particulier lié à la munificence des souverains maures qui voulaient, à l'imitation de César ou d'Auguste, donner dans leur capitale des venationes et des combats entre troupes armées. Les techniques de construction permettent de dater de la même époque l'enceinte de la ville et l'aqueduc qui l'alimentait en eau, du moins dans son état primitif. L'enceinte de Caesarea entre ainsi dans la série des très vastes enceintes, celles qu'on connaît en particulier en Gaule, à la 
même époque ; comme celles-ci d'ailleurs, elle n'entourait pas un espace entièrement bâti: seuls les 150 ha de la plaine littorale furent effectivement occupés par des constructions continues. Un sondage pratiqué dans la partie occidentale de la ville a montré que le réseau géométrique des rues y remontait à l'époque royale.

L'étude archéologique de la ville reste encore largement à faire. La chronologie des édifices énumérés est imprécise. Ainsi il est bien difficile de proposer une date pour le cirque. Beaucoup d'autres édifices sont inconnus. On ignore où était le forum de la ville de Juba ; car il y en eut certainement un. P. Pensabene a souligné l'importance des séries augustéennes dans les chapiteaux et pilastres de marbre. Tous les éléments d'entablement conservés sur la place des Martyrs, au centre de la ville, ou au Musée, ne proviennent pas du théâtre; eux aussi comportent des séries augustéennes. Les uns et les autres constituent une preuve de la splendeur de la ville construite par Juba. Si donc dans le détail la discussion est largement ouverte, s'il est possible que des édifices projetés par Juba n'aient été construits ou achevés que beaucoup plus tard, l'ampleur de l'urbanisme royal est une réalité fondamentale maintenant bien établie qui rend d'ailleurs en partie compte de la médiocrité relative des témoignages d'activité édilitaire portant sur les périodes ultérieures. Les successeurs des rois eurent sans doute longtemps pour souci principal d'achever et de maintenir en état le patrimoine monumental qui leur avait été légué. Cette phase majeure de l'histoire de la ville vit également les débuts de la romanisation juridique des notables maures reconnaissables à leur gentilice Iulius, aux côtés desquels prennent place les descendants d'émigrés romains ou italiens.

9 Si la plupart des constructions importantes du $\mathrm{I}^{\mathrm{er}}$ siècle paraissent devoir être mises au crédit des rois maures, l'époque claudienne joue un rôle décisif dans la mise en place du cadre juridique. La ville est promue au rang de colonie et sans doute la plupart de ses notables reçoivent-ils la citoyenneté romaine. Une telle promotion est à la fois la conséquence d'une romanisation ancienne et la récompense probable de son calme pendant la guerre contre Aedemon qui prétendait venger son maitre, le roi Ptolémée. Caesarea conserve sa fonction de capitale ; à l'administration royale se substituent un gouverneur romain qui est un procurateur équestre et son officium. Ultérieurement, le corps civique et son élite politique, l'ordo, ne paraissent avoir été renforcés que par la promotion interne de quelques familles nouvelles descendant soit d'affranchis impériaux soit d'affranchis de grandes familiae de l'aristocratie romaine dont ils avaient pu être les agents locaux. La structure sociale de la population aux $\mathrm{I}^{\mathrm{er}}$ et $\mathrm{II}^{\mathrm{e}}$ siècles est relativement bien connue par un grand nombre d'épitaphes recueillies dans les nécropoles urbaines. Pendant la période allant d'Auguste à Trajan, plus de la moitié des personnes nommées sur les épitaphes sont probablement d'origine ou de condition servile. Dans celle qui suit, esclaves et affranchis ne représentent plus que $5 \%$ des personnes nommées sur les épitaphes et les porteurs de noms uniques sont moins de 13 $\%$. Cette structure sociale connue par l'épigraphie différencie Caesarea des autres villes d'Afrique du Nord romaine dont la population paraît avoir compté une faible proportion d'esclaves. Mais une telle situation est normale car le développement de Caesarea est contemporain de la fin de l'apogée du système esclavagiste dans l'Empire romain et la ville est importante dès le $\mathrm{I}^{\text {er }}$ siècle av. J.-C, alors que l'essor des autres villes de Maurétanie et même d'une grande partie de la Proconsulaire date du siècle suivant. En ce sens Caesarea peut être rapprochée d'une autre capitale africaine, Carthage. L'étude sociale confirme donc l'étude archéologique de la ville: l'une et 
l'autre mettent en évidence la précocité (relative évidemment à ce secteur provincial) du développement urbain de Caesarea. En revanche, la permanence de l'habitat sur le site, la réutilisation des inscriptions urbaines dans les constructions et l'absence de fouilles systématiques du centre monumental expliquent la médiocre connaissance que nous avons d'une élite municipale qui paraît avoir été plus riche et plus puissante que ne le laisse penser l'épigraphie locale. Elle laisse en effet mal entrevoir la part prise par l'aristocratie locale à l'administration de l'Empire. Mais on sait qu'en la personne du Macrin, Caesarea donna un empereur à Rome.

Villae et agglomérations paysannes dépendant de Caesarea de Maurétanie

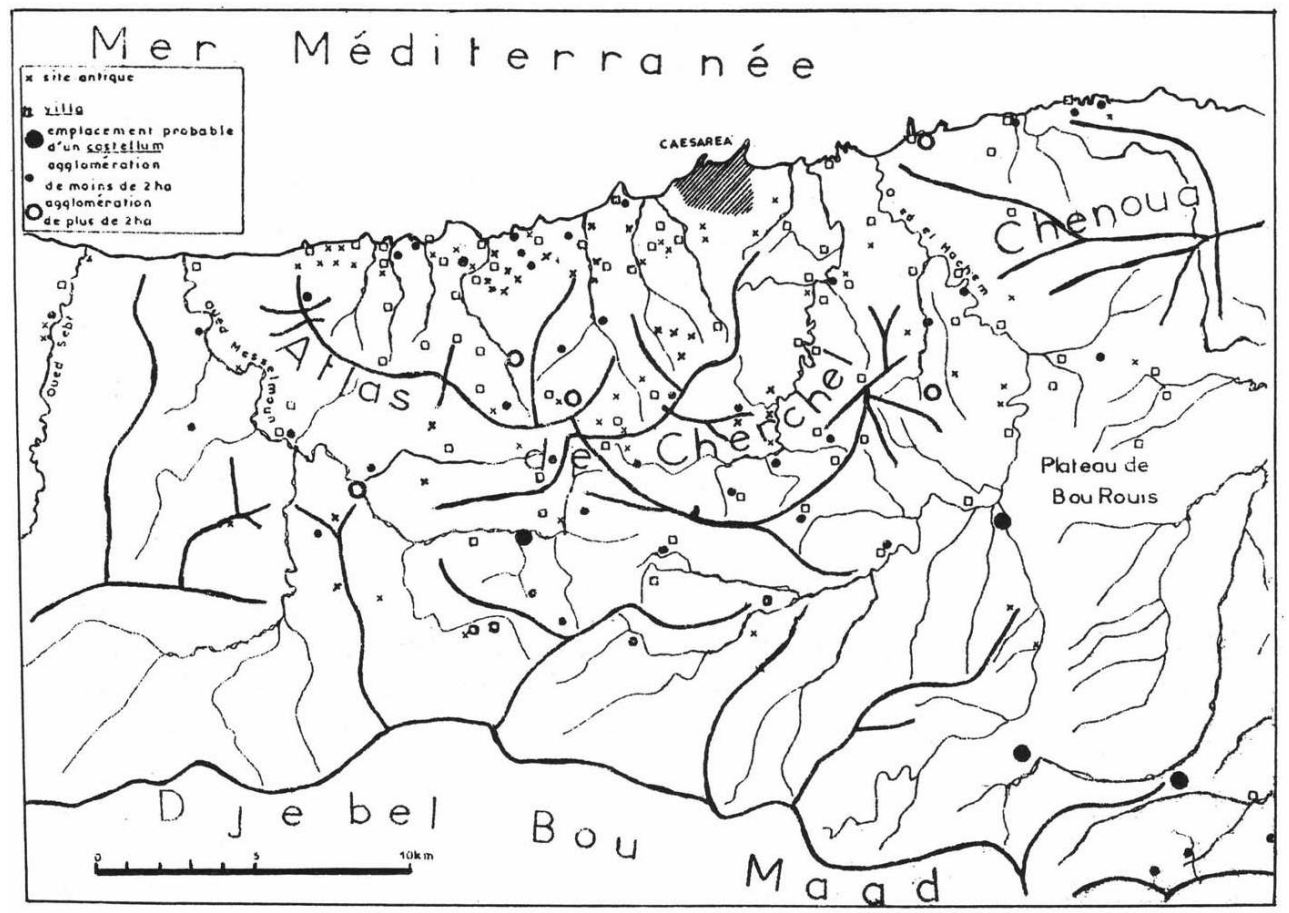

10 L'époque augustéenne avait été le grand moment de l'urbanisme de Caesarea. L'étape décisive de sa romanisation juridique se situe à l'époque claudio-néronienne. C'est sous les Flaviens qu'elle semble s'être dotée de l'assise économique nécessaire à sa survie en organisant de manière systématique l'exploitation agricole de son territoire. Il ne s'agit évidemment pas d'une création ex nihilo. Plusieurs établissements agricoles dont la villa du Nador fouillée entre 1972 et 1974 par une mission algéro-italienne, existaient à l'époque royale. Les campagnes étaient cultivées bien avant la mise en chantier de la ville romaine. Mais les habitats ruraux correspondant à la Iol pré-romaine sont difficilement identifiables tant que l'on ne connaîtra pas mieux les céramiques modelées indigènes. Les villae romaines, en revanche, établissements agricoles dont le mode de construction et le plan sont caractéristiques et que des tessons abondants permettent de dater, ont pu être étudiées et cartographiées. Elles occupent, autour de Caesarea, un espace semi-circulaire d'une quinzaine de kilomètres de rayon: au nord, du côté de la mer, un espace défini archéologiquement par la présence de villae régulièrement espacées sur le plateau littoral et dans les vallées et réparties dans les vallons en berceaux dégagés par l'érosion sous les lignes de crêtes de l'Atlas de Cherchel ; sur les marges, une seconde zone définie par l'absence de villae. Mais partout à côté des villae existe un habitat qui constitue la donnée permanente du paysage rural : 
de petites fermes ou cabanes, isolées ou réunies en agglomérations autonomes ou regroupées autour des villae. En l'absence de fouilles systématiques des villae, les cultures sont bien mal connues. Nul doute que les cultures vivrières de base, et parmi elles la céréaliculture, n'aient occupé une place essentielle. Mais les vestiges de surface permettent d'identifier deux cultures particulières. A proximité de la ville, de nombreux restes de réservoirs destinés à l'irrigation sont à mettre en rapport avec les jardins fournissant les légumes à la ville. Sur l'ensemble de l'espace rural, une prospection de surface a permis d'identifier une grande quantité de vestiges d'huileries : de grandes huileries regroupant probablement plusieurs dizaines d'unités de pressage, qui sont en réalité des villae spécialisées ; des huileries de villae comportant trois ou quatre pressoirs regroupés dans un quartier de la ferme; des huileries à pressoir unique dispersées dans la campagne près de restes de cabanes ou dans une agglomération.

11 Villae spécialisées et villae à fonctions diversifiées relèvent d'une même forme d'exploitation de la terre, tandis que les huileries à pressoir unique renvoient à une autre. Or on constate que les grandes huileries spécialisées, dont les plans sont d'ailleurs identiques, ont une durée d'existence bien particulière allant de la fin du I ${ }^{\mathrm{er}}$ siècle ap. J.-C. à celle du $\mathrm{III}^{\mathrm{e}}$ siècle ap. J.-C, alors que les tessons associés aux huileries à pressoir unique montrent que celles-ci ont fonctionné durant toute l'Antiquité. On peut en conclure qu'une même culture a fait l'objet de deux modes différents d'exploitation : l'une sous forme microfundiaire a duré toute l'Antiquité, l'autre, sous forme latifundiaire, est plus particulière aux $\mathrm{II}^{\mathrm{e}}$ et $\mathrm{III}^{\mathrm{e}}$ siècles.

L'ampleur des créations urbanistiques à Caesarea dans la première moitié du $\mathrm{I}^{\mathrm{er}}$ siècle ap. J.-C. explique que l'activité édilitaire des siècles suivants n'apparaisse pas toujours avec évidence. Pourtant le second siècle vit la construction des grands établissements thermaux, en particulier les thermes de l'ouest, le mieux connu et le plus important des établissements thermaux de la ville; son plan le situe à une époque où les architectes romains maîtrisaient parfaitement les problèmes posés par la diversité des opérations de bain. Ces thermes, dont le plan rappelle celui des thermes de Timgad, mesuraient $115 \mathrm{~m}$ sur $70 \mathrm{~m}$. C'est peut-être au second siècle que fut construit (ou achevé ?) le cirque dans le quartier occidental de la ville. Les monuments augustéens furent régulièrement entretenus et même améliorés ou transformés. Les travaux les plus notables concernent le rempart de la ville, l'aqueduc oriental et sans doute aussi les édifices liés aux jeux, l'amphithéâtre qui fut agrandi et le théâtre dont le dispositif de représentation fut modifié. Il est sûr que les troubles qui, au second siècle, et plus particulièrement à l'époque antonine, affectèrent la Maurétanie, n'eurent guère de conséquences sur le développement urbain. A cette époque, il s'agit encore des réactions violentes de tribus limitées et spoliées et non d'une révolte massive. La première partie $d u$ III $^{\mathrm{e}}$ siècle se situe dans la parfaite continuité de la période précédente. De l'époque sévérienne datent la porta triumphalis du cirque et, comme viennent de le montrer les fouilles algéro-britanniques, la construction d'un forum (nouveau?) au centre de la ville. La population urbaine est profondément romanisée. Le contrôle militaire de Rome est également à son apogée et un certain nombre des unités auxiliaires stationnées à Caesarea sont parties vers la nova praetentura plus au sud. 
13 A quel chiffre peut-on évaluer la population urbaine à son apogée? Des chiffres considérables ont été proposés : $\mathrm{S}$. Gsell parlait d'une centaine de milliers d'habitants et $\mathrm{y}$ voyait la seconde ville d'Afrique après Carthage. $C$.

14 Courtois situait Caesarea au niveau de Lepcis Magna et proposait le chiffre de 37000 habitants. Pour ma part, je trouve le chiffre encore excessif et je ne pense pas que la ville ait jamais eu plus de 20 à 22000 habitants. En effet sur les 370 ha enclos par l'enceinte, seuls les 150 ha situés en dessous de la courbe de niveau des $50 \mathrm{~m}$ ont été effectivement occupés mais il faut encore tenir compte de l'importance des espaces non bâtis ou occupés par des monuments, en particulier par le cirque, l'amphithéâtre et peut-être même par un stade.

Au-delà de cette période, l'histoire de la ville pose de difficiles problèmes. D'un côté, nous constatons l'abandon au $\mathrm{IV}^{\mathrm{e}}$ siècle d'un grand nombre des villae qui paraissent assurer l'assise économique des élites urbaines; Ammien Marcellin décrit en termes catastrophiques la situation de Caesarea, "ville naguère opulente et célèbre " (Histoire, XXIX, 5, 18), prise et complètement brûlée en 371 par le prince maure révolté Firmus; la documentation épigraphique devient rare. D'un autre côté une lettre de Symmaque appuyant une demande de moratoire fiscal déposée par l'évêque Clément de Césarée, donne l'image de notables disposant d'un bon réseau de relations et encore riches même s'ils se disent incapables de prendre à leur charge le remboursement des contributions de toute la province sur lesquelles Firmus avait fait main basse (Symmaque, Lettres, I, 64); les fouilles des années 1960 ont mis au jour un nombre important de riches domus datant justement du IV siècle. Les modifications du théâtre, transformé en amphithéâtre qui pouvaient être interprétées non comme un témoignage sur l'appauvrissement de la ville, sont, peut-être plutôt le résultat d'une évolution des goûts en matière de spectacles. 


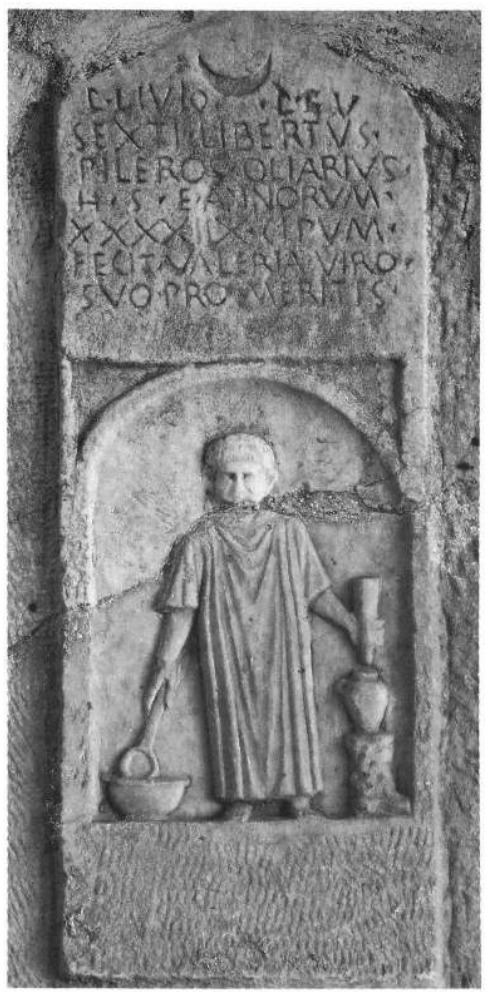

$\mathrm{Au}$ IV $\mathrm{V}^{\mathrm{e}}$ siècle, Caesarea devient chrétienne. Quelques découvertes d'églises et d'inscriptions montrent que le christianisme pénètre les campagnes. Mais les rares documents dont on dispose ne permettent pas d'écrire l'histoire de ces modifications religieuses ni d'apprécier l'incidence du triomphe de la religion nouvelle. Ce qui est sûr, l'appui prêté par le grand seigneur païen Symmaque à l'évêque Clément le montre, le décor des belles domus le confirme, c'est que passée l'époque des persécutions, les deux religions coexistent au sein d'une même culture. En définitive, les seules données précises sur le christianisme local concernent l'écho laissé, comme dans beaucoup de villes africaines, par les querelles liées au schisme donatiste: en 411, l'Église de Caesarea était partagée entre deux évêques, le donatiste Emeritus et le catholique Deuterius. En 418, saint Augustin vint y prêcher sans grand succès. Au-delà, nos connaissances s'estompent. En dehors de l'histoire administrative de la province dont Caesarea était la capitale, on ignore presque tout de l'évolution de la ville du ve siècle jusqu'à la fin du Moyen-Age : quelle fut l'incidence de la conquête Vandale après 455 ? Quelle était son importance réelle lorsque la reconquête de Bélisaire en 534 en fit la capitale de la Maurétanie Seconde et la résidence d'un gouverneur et d'un duc? A la fin $\mathrm{du} \mathrm{VI}{ }^{\mathrm{e}}$ siècle, elle semble perdue par Byzance, car elle a disparu des listes de Georges de Chypre composées au début du viI siècle et le duc s'était transporté à Sétif. Peut-être le trésor byzantin de sous d'or trouvé sous la place de l'Église était-il en relation avec les troubles qui durent marquer les passages de la ville sous le contrôle du royaume maure qui se reconstitue à l'intérieur. Les fouilles effectuées à l'emplacement du forum sévérien par une mission archéologique algéro-britannique ont montré que le forum, la basilique civile et une basilique chrétienne sont restés en usage jusqu'au début du $\mathrm{VI}^{\mathrm{e}}$ siècle. Par la suite, le site n'est pas abandonné mais ce secteur ne fait l'objet d'aucun réaménagement monumental. 


\section{BIBLIOGRAPHIE}

On trouvera une bibliographie exhaustive du site de Caesarée dans LEVEAU Ph., Caesarea de Maurétanie. Une ville romaine et ses campagnes, collection de l'École française de Rome, $n^{\circ} 70,1984$, $556 \mathrm{p}$.

BENSEDDIK N. et POTTER T.-W., Fouilles du forum de Cherchel, Alger, 1986, $4^{e}$ supplément au Bulletin d'Archéologie algérienne.

DUVAL N., Une petite église chrétienne sur le forum de Cherchel, Rev. des Études augustiniennes, 34, 1988, p. 247-256.

DUVAL P.-M., Cherchel et Tipasa ; recherches sur deux filles fortes de l'Afrique romaine, Paris, 1946.

GOLVIN et LEVEAU Ph., L'amphithéâtre et le théâtre-amphithéâtre de Cherchel ; monuments à spectacles et histoire humaine à Caesarea de Maurétanie, MEFRA, 91, 2, 1979, p. 817-843.

GSELL S., 1952, Cherchel, antique Iol-Caesarea, mis à jour par M. Leglay et E.-S. Colozier, Alger.

GSELl S., Cherchel. Dans Chabrol F. et LECLERCQ H., Dictionnaire d'archéologie chrétienne et de liturgie, t. 3, $1^{\text {re }}$ partie, Paris, 1948, col. 1269-1281.

MARCHAND H., Cherchel préhistorique. Bull, de la S.P.F., 10, 1932, p. 1-7.

PENSABENE P., Les chapiteaux de Cherchel. Étude de la décoration architectonique (1982), $3^{\mathrm{e}}$ supplément, Bull. d'Archéologie algérienne, Alger, 1982.

PICARD G. Ch., La date du théâtre de Cherchel et les débuts de l'architecture théâtrale dans les provinces romaines d'Occident, C.R.A.I., 1975, p. 386-397.

INDEX

Mots-clés : Algérie, Antiquité, Villes 